\title{
TLR9-based immunotherapy for the treatment of allergic diseases
}

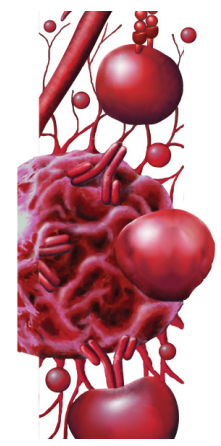

Toll-like receptors (TLRs), a family of pattern recognition receptors expressed on many cell types of innate immunity, recognize the pathogen-associated molecular patterns of microbes. The hygiene hypothesis suggests that a reduced microbial exposure in early childhood increases the susceptibility to allergic diseases due to deviation in development of the immune system. TLRs are key roles in the right and healthy direction of adaptive immunity with the induction of T-helper 2 toward Th1 immune responses and regulatory T cells. TLR ligand CpG-ODN-based immunomodulation is independent of allergen and it mainly affects innate immune system. While, CpG-oligodeoxynucleotide-based vaccination is allergen specific and induces adaptive immune system. The use of agonists of TLR9 in two distinct strategies of immunotherapy, immunomodulation and vaccination, could be presented as the curative method for the treatment of allergic diseases.

First draft submitted: 29 August 2016; Accepted for publication: 8 February 2017; Published online: 17 March 2017

Keywords: allergic diseases $\bullet$ immunotherapy • TLR9 • Toll-like receptors

The incidence of allergic diseases is increasing globally and has reached a point to be a costly public health issue, especially in industrialized countries [1]. The probable explanation is the 'hygiene hypothesis', which implies an inverse relationship between increasing allergic diseases and the lack of exposure to microbes during early childhood [2]. Therefore, allergens play the major role in creating allergic diseases and inhaled or food allergens could cause severe reactions such as anaphylactic shock in some people [3]. The immunopathology of these diseases result from $\mathrm{T}$ helper 2 (Th2) cells immune responses against harmless environmental antigens. The main role of Th2 cells is to produce IL-4 and IL-5, which can develop allergic reactions [4]. Also, IL-4 promotes differentiation of naive $\mathrm{TH} 0$ cells to $\mathrm{TH} 2$ phenotype and stimulates $\mathrm{B}$ cells to secrete IgE. Additionally, IL-5 increases the development of eosinophils from progenitor cells [5].
In allergic diseases in addition to the increase in Th2 activity, Th1 cell type cytokine secretion will be ameliorated [6]. It has been shown that exposure to bacterial and viral infections in childhood would enhance the immune system toward Th1 cells [7]. Therefore, the increasing incidence of allergic diseases could be associated with the decrease in infections during childhood.

Toll-like receptors (TLRs) play an essential role in the early innate immune response against microbial pathogens $[8,6]$. TLRs express on the surface or in the vacuoles of cell subsets which are involved in the host defense such as neutrophils, natural killer cells (NKCs) and antigen-presenting cells (APCs). Different component of pathogens inducing TLR signaling are called pathogenassociated molecular patterns. Also, TLRs trigger dendritic cells (DCs) maturation which induces the expression of costimulatory molecules and secretion of inflammatory
Shokrollah Farrokhi', Narjes Abbasirad ${ }^{1}$, Ali Movahed ${ }^{2}$, Hossein Ali Khazaei $^{*}, 3$, Masoud Pishjoo 4 \& Nima Rezaei ${ }^{5}$

'Department of Immunology, Asthma \& Allergy, The Persian Gulf Tropical Medicine Research Center, Bushehr University of Medical Sciences, Bushehr, Iran

${ }^{2}$ Department of Biochemistry, Bushehr University of Medical Sciences, Bushehr, Iran

${ }^{3}$ Clinical Immunology Research Center, Department of Immunology \& Hematology, Zahedan University of Medical Sciences, Zahedan, Iran ${ }^{4}$ Clinical Immunology Research Center, Zahedan University of Medical Sciences, Zahedan, Iran

${ }^{5}$ Research Center for Immunodeficiencies, Tehran University of Medical Sciences, Tehran, Iran

*Author for correspondence:

Tel.: +989153403094

Fax: +985433295571

h_khazaei118@yahoo.com 
cytokine such as TNF- $\alpha$, IL-1, IL-6 and IL-12. These cytokines induce Th1 immune responses and prevent differentiation of Th 2 cells [7]. Additionally, it has been known that TLRs express on regulatory $\mathrm{T}$ cells, which then play an important role in the modulation of immune system [7]. In conclusion, it has been proposed that TLRs play an important role in reducing Th2mediated allergic responses and prevent the development of allergic diseases [7]. It is suggested that TLRs can be used for the treatment of allergic diseases.

\section{Toll-like receptors}

TLRs are highly conserved pattern recognition receptors in vertebrates and insects and play a significant role in activation of the innate immune system. A class of type I transmembrane proteins were identified in Drosophila, which are important in defense against infection $[9,10]$. The members of TLRs have two important domains; the extracellular which consists a leucine-rich region, and has an important role in binding to the ligands and the intracellular domain with approximately 200 amino acids. The similarity of this latter region with IL-1 receptor called Toll/IL-1R (TIR) domain makes it necessary for the downstream signaling. These receptors are expressed mainly on macrophages and DCs which recognize specific microbial structures called pathogen-associated molecular patterns [11,12]. The TLRs including TLR1, 2, 4, 5, 6 and 11 are expressed on the surface of cells and TLR3, 7, 8 and 9 which recognize nucleic acid are expressed in the cells [13].

TLRs recognize specific ligands that form a part of components of microbial pathogens as part of component of microbial pathogens. Signal transduction happens by these receptors which lead to activation of adaptor proteins and the transcription factors including NF- $\mathrm{KB}$ and activator protein (AP)-1. These factors trigger inflammatory responses and interferon regulatory factor (IRFs), which finally create antiviral responses leading to eradication of pathogens [14]. The heterodimers TLR1/TLR2 and TLR2/TLR6 recognize various bacterial components including peptidoglycan, lipoproteins and lipoarabinomannan of mycobacteria and zymosan of yeast [14-17]. Moreover, TLR3, TLR8 and TLR9 express the intracellular vacuoles that detect dsRNA, ssRNA of many viruses and bacterial unmethylated CPG DNA, respectively [14,18,19,20,21,22]. Also, lipopolysacaride of Gram-negative bacteria and lipotichoic acids of Gram-positive bacteria are recognized by TLR4 and TLR5 recognizes bacterial flagellin [23].

TLRs utilize different adapter proteins such as MyD88, TIR-associated protein (TIRAP), MyD88 adaptor-like protein, TIR domain-containing adaptor protein-inducing IFN- $\beta$ (TRIF) and TRIF-related adaptor molecule. All the TLRs utilize MyD88 as an adaptor protein in their signaling pathway except TLR3 which utilizes TRIF. TRIF-related adaptor molecule and TIR-associated protein are adaptor proteins that are used by TLR4 [14].

According to the research conducted in the field of molecular immunology, TLRs are as novel targets in the prevention and treatment of allergic diseases $[9,24,25]$. The involvement of TLRs trigger a cascade of cell signaling that leads to the production of Th1-related cytokines such as IL-12 and IFN- $\gamma$. These cytokines modulate allergic responses by prevention of IL- 4 and IL-5, which are secreted by Th2 lymphocytes [26]. In this review the method of TLR9 immunotherapy in the treatment of allergic diseases will be discussed.

\section{Natural \& synthetic ligands of TLR9}

TLR9 is one of the members of the TLRs family expressed dominantly in B lymphocytes and plasmacytoid DCs $\left({ }_{\mathrm{p}} \mathrm{DCs}\right)$. It is located in the endoplasmic reticulum, endosome and lysosome [27,28]. This glycoprotein has an extracellular leucin-rich and cytoplasmic signaling domain [29]. TLR9 recognizes unmethylated CpG dinucleotides which are common in bacterial and viral genomes and relatively rare $(\sim 1 \%)$ in vertebrate genomes [30]. In prokaryotes DNA, methylation occurs rarely and cytosines are unmethylated in comparison with vertebrate genomes [30,31]. Not only natural DNAderived bacteria and viruses, but also synthetic oligodeoxynucleotides (ODN) containing unmethylated CpG motifs are ligands for TLR9 [32]. Activation of TLR9 by CPG DNA or ODN leads to induction of strong Th1 immune response. Th1 produces cytokines such as IL-12, IL-18 and IFN- $\gamma$, which continues to activate the NKC and enhances CD8 T-cell response. Finally, these activities of Th1 inhibit Th2-type immune response and cytokines production of IL- 4 and IL-5 [33].

CpG-ODNs are also called immunostimulatory sequence-ODN (ISS-ODN) [26]. ISS-ODNs contain unmethylated $\mathrm{CpG}$ dinucleotides within a palindromic hexamer having the formula: 5'-purin-purin-CpGpyrimidin-pyrimidin-3' [34]. There are five classes of CpG-ODNs based on their sequence, secondary structures and effect on human peripheral blood mononuclear cells (PBMCs) which contain classes of A (Type D), B (Type K), C, P and S [32,35,36].

Class A has potential for inducing IFN- $\alpha$ production by $\mathrm{DC}$. Its backbone structure is mainly a phosphodiester with a phosphorothioate at one or both ends of ODN [37,38]. Class B strongly activates B cells proliferation and production of antibody [30]. Class $\mathrm{C}$ has the mechanism of action similar to class A and B $[39,40]$. Phosphorothioate-ODN mainly activates $\mathrm{B}$ cell and up 
to less extent activates macrophages or NKCs. On the contrary, phosphodiester-ODN is a potent activator for macrophages or NKCs rather than B-cell proliferation (Table 1) [30].

\section{TLR9 signaling}

TLR9 signaling consists of two distinct pathways: the myD88-dependent and myD88-independent pathways [41]. Stimulation of TLRs by their ligands leads to the activation of transcription factor such as AP-1, NF-KB and IRFs [41]. The myD88-dependent pathway leads to the production of inflammatory cytokines such as IL-6, IL-12P40 and TNF while the myD88-independent pathway mediates type I IFN response [41]. Binding of TLR9 to its ligand leads to conformational changes in cytoplasmic TIR domain resulting in the recruitment of adaptor proteins including myD88 which is essential for initiating TLR9 signaling [35]. Upon recruitment of myD88 to TIR domain, it interacts with interleukin-receptor associated kinase-4 (IRAK4), and subsequently causes phosphorylation of IRAK4 which leads to activation of IRAK1 [42,41]. After the separation of IRAK1 and IRAK4 from myD88-TLR complex, they activate TNF receptor associated factor (TRAF-6) [41]. Activation of TRAF- 6 leads to the activation of TGF- $\beta$ activated kinase 1 (TAK1) and TAK1-binding proteins (Tables $1 \&$ 2). TAK1 activates the IкB kinase (IKK) complex (IKK $\alpha$, IKK $\beta$ and NEMO/IKK $\gamma$ ). IKK complex phosphorylates IKB which subsequently activates $\mathrm{NF}-\kappa \mathrm{B}$, inducing production of inflammatory cytokines such as TNF, IL-1 and IL-12 [43]. Moreover, IRAK1 phosphorylates and activates IRF7 causing the induction of type I IFNs which are essential for the defense against viral infections (Figure 1) $[43,44]$.

\section{CpG-immunonomadulation versus vaccination}

CpG (ISS)-ODN induces Th1-based immune response and produces IFN- $\gamma$ and IgG2a which prevents the development of Th2-mediated hypersensitivity in mouse models of allergic diseases [34,45]. Therefore,
ISS-ODNs could be effective in the treatment of allergic diseases [35]. There are two distinct strategies for the use of ISS-ODN in the treatment of allergic diseases: immunomodulation and vaccination [34]. ISS-ODN based immunomodulation is independent of allergen and it mainly affects innate immune system leading to the activation of NKC, MQ and DC. The therapeutic effects of ISS-ODN based immunomodulation develop rapidly and persist for a short term (for weeks). While, ISS-ODN based vaccination is allergen specific and induces adaptive immune system. Its effects in the treatment of allergic diseases develop slowly with longterm duration that remains for at least 1 year [34,46]. Consequently, both the ISS-ODN based immunomodulation and vaccination are effective in the treatment of allergic diseases.

Moreover, studies have shown that ISS-ODN based vaccination can prevent the immediate hypersensitivity response and late-phase allergic reaction [45]. Vaccination with ISS-ODN was done in two different strategies: ISS-ODN mixed and conjugated allergens (AIC) [46]. Both the methods shift Th2 toward Th1 immune response, which then leads to the treatment of allergic diseases [46]. However, studies have shown that AIC improves immunogenicity and reduces allergenicity in comparison with mixed allergens $[46,47]$. Due to colocalization of ISS-ODN and allergen on APCs, uptake and presentation of allergen by APCs will be facilitated and leads to increase immunogenicity with AIC [48]. Conjugation of allergen to ISS-ODN masks allergen epitopes thereby, prevents recognition of epitopes by specific $\operatorname{IgE}$ which finally leads to reduction in allergenicity by AIC [39]. In addition, AIC induces Th1based immune responses such as IFN- $\gamma$ and IgG2a. Also, it reduces IL-5 response compared with allergen mixed ISS-ODN, significantly (Tables $2 \& 3$ and Figure 2) [34,45].

Immunotherapy based CpG-ODN leads to induction of Th1, regulatory $\mathrm{T}$ lymphocytes response and activation of innate immune system which reduces Th2 response [26]. Therefore, binding of CpG-ODN to TLR9 produces IL-12 and IL-10 by DCs leading to

Table 1. Comparison of the effects of the different classes of CpG-CpG-oligodeoxynucleotide.

\begin{tabular}{|llll|}
\hline ODN classes & IFN- $\alpha$ secretion & B-cell proliferation & PC maturation \\
\hline A-ODN & +++ & + & ++ \\
\hline B-ODN & + & +++ & +++ \\
\hline C-ODN & ++ & +++ & +++ \\
P-ODN & ++ & + & ++ \\
\hline S-ODN & - & - & \\
\hline +: week effect, ++: moderate effect, +++: strong effect. & & \\
$\begin{array}{l}\text { ODN: Oligodeoxynucleotide; } D C: \text { Plasmacytoid dendritic cell. } \\
\text { Data taken with permission from [37]. }\end{array}$ & & \\
\hline
\end{tabular}




\begin{tabular}{|c|c|c|}
\hline & Immunomodulation & Vaccination \\
\hline Targeting & Innate immunity & Adaptive immunity \\
\hline Effects duration & Short-term (weeks) & Long-term (months/years) \\
\hline Type of cell type effects & NK, MQ, DC & B cell, Th1 \\
\hline Cytokine induction & IFN- $\alpha / \beta / \gamma$, IL-12, IL-6, IL-18, $\downarrow$ IL-4 receptor & IFN- $\gamma$, IL-10, IL-4, $\downarrow$ IL-5, IL-13 \\
\hline
\end{tabular}

the induction of Th1 and regulatory $\mathrm{T}$ lymphocytes responses [49].

It has been shown that CpG-ODN induces the production of indoleamine 2,3-dioxygenase by DCs [26,50]. This enzyme enhances the number and function of T-regulatory lymphocytes [52]. Therefore, immunotherapy with $\mathrm{CpG}-\mathrm{ODN}$ induces T-regulatory cells and indoleamine 2,3-dioxygenase leading to the suppression of Th2 responses and so clinical manifestation of allergic diseases [49,51]. Based on the above information, CpG-ODN immunotherapy could be used in the treatment of allergic diseases.

\section{CpG-ODN immunotherapy in animal models}

\section{Allergic rhinitis}

Different studies have shown the potentials of CpGODN immunotherapy in the treatment of allergic rhinitis in animal models $[52,53]$. Hussain $e t$ al. showed that CpG-ODN immunotherapy in mice model of allergic rhinitis sensitized with OVA markedly decreased nasal irritation and eosinophil count in their airways [52]. Also, Rhee $e t$ al. showed that treated mice with CpG-ODN decreased release of histamine and nasal eosinophilic

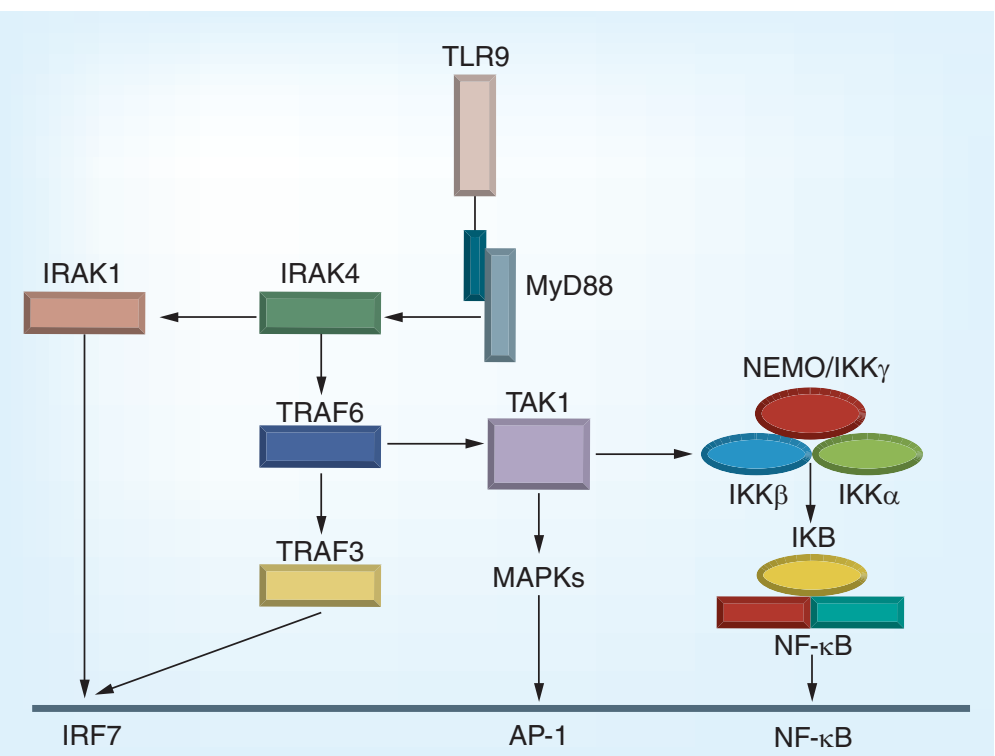

Figure 1. Schematic Toll-like receptor 9 signaling pathway. inflammation [53]. Moreover, immune responses of the mice shifted from Th2 toward Th1 cells. In the same investigation they showed that the efficacy of $\mathrm{CpG}$ ODN in reducing allergic inflammation was more than dexamethasone [53].

\section{Asthma}

Based on studies conducted on murine models of asthma, it has been shown that CpG-ODN immunotherapy could reduce Th2-based responses leading to improvement in manifestation of asthma [54-56]. Kline et al. reported that CpG-ODN immunotherapy in C57BL/6 model of asthma which was sensitized to soluble egg antigen from the schistosome, decreased eosinophilia and bronchial airway infiltration of allergic inflammatory cells [54]. CpG-ODN immunotherapy also increased Th1 cytokines such as IL-12 and IFN- $\gamma$ and attenuated manifestations of asthma in C57BL/6 model [54].

The results from another study showed that ISSODN immunotherapy in ragweed sensitized mice inhibited production of Th2 cytokines and suppressed the rise in specific IgE after allergen challenge [55]. Also, Santeliz et al. reported that administration of CPG-ODN in murine model of asthma induced Th1 immune response. Additionally, they showed that CpG-ODN conjugated allergen was more effective than CpG-ODN mixed with allergen in attenuation of Th2-driven allergic airway responses [56]. Moreover, it has been shown that administration of AIC in mice model of asthma induced IFN- $\gamma$ and decreased IL- 4 and IL-5 [57].

\section{Other allergic disorders}

CpG-ODN immunotherapy has been investigated as a new therapeutic method for the treatment of other allergic disorders such as conjunctivitis, airway remodeling and anaphylactic shock. In murine model of allergic conjunctivitis induced by ragweed, CpGODN immunotherapy considerably reduced the level of ragweed-specific $\mathrm{IgE}$ and late-phase infiltration of allergic cells [58]. Also, Cho et al. investigated the effects of $\mathrm{CpG}-\mathrm{ODN}$ on the airway remodeling. They 
Table 3. The effects of three methods immunotherapy on the production of some cytokines.

\begin{tabular}{|llll|}
\hline Immunotherapy by & IFN $-\gamma$ & IgG2a & IL-5 \\
\hline Allergen only & + & + & +++ \\
\hline Allergen-ISS conjugate & +++ & +++ & +++ \\
\hline Allergen-ISS mix & ++ & ++ & \\
$\begin{array}{l}+ \text { : week effect, }++ \text { : moderate effect, +++: strong effect. } \\
\text { ISS: Immunostimulatory sequence. }\end{array}$ & & \\
\hline
\end{tabular}

found that CpG-ODN immunotherapy in sensitized mice with allergen reduced the level of TGF- $\beta$ as a profibrotic cytokine. Peribronchial fibrosis and mucous production was inhibited in these mice. Therefore, immunotherapy with CpG-ODN inhibits TGF- $\beta$ production and airway remodeling [59]. Similarly, Fanuchi and colleagues have reported that CpG-ODN immunotherapy reduced eosinophilic inflammation and prevented airway remodeling in a model of allergic monkey [60].

Importantly, it was found that administration of OVA conjugated CpG-ODN in mice model of anaphylactic shock, increased Th1 dominant immune response which causes a decrease in $\mathrm{IgE}$ level and protected OVA sensitized BALB/c mice from anaphylactic shock [61].

\section{CpG-ODN immunotherapy in human clinical trials}

\section{Allergic rhinitis}

Results of an investigation by Creticos et al. showed that immunotherapy using conjugated ISS-ODN and Amb a1 (AIC) in adults with allergic rhinitis (AR) reduced acute allergic responses. Also, AIC increased Amb al specific IgG but reduced the Amb a1 specific IgE. Therefore, they suggested that AIC immunotherapy could be used for the treatment of ragweed AR [62]. In another study by Asai et al. it was found that immunotherapy with AIC in subjects with AR after seasonal ragweed-pollen exposure increased $\mathrm{CD} 4^{+} \mathrm{CD} 25^{+} \mathrm{T}$ regulatory cell in the nasal mucosa [63]. Our previous study was performed to investigate the effect of CpG-ODN in alteration of T-helper (Th)1/Th2 balance of patients with AR treated with intranasal corticosteroids and antihistamines. PBMCs of patients with AR were isolated before and after 45 days therapy. Cytokine production and specific Ch.a IgE in response to $\mathrm{CpG}$ co-administration of natural chenopodium album (CpG/Ch.a) or recombinant Ch.a (CpG/rCh.a) allergen were investigated in supernatants of cultured PBMCs using ELISA. Intracellular IL-10 was also assessed in $\mathrm{CD}^{+}$ cells using flow cytometry. The results of our study showed that treatment of PBMCs of patients with chenopodium album AR by CpG ODN mixed allergen induced IFN- $\gamma$ and IL-10 while suppressed IL- 4 and IL-13 [64]. Moreover, Tulic et al. investigated the effect of AIC in ragweed-sensitive patients with AR. They examined the role of AIC on eosinophilia and cytokine mRNA expression in the nasal mucosa of the two groups (AIC and placebo-treated patients). The results of the above study showed that after 4 to 5 months AIC immunotherapy, eosinophilia and IL-4 mRNA-positive cells were significantly decreased. Additionally, the number of IFN- $\gamma$ mRNA-positive cells was increased in AIC-treated patients as compared with the placebo. Also, the allergic symptoms of AIC-treated patients were decreased [65].

\section{Asthma}

It has been shown that A-type of CpG-ODN in virus-like particles package was effective in reducing symptoms of AR and asthma [66]. Also, Gauvreau et al. reported that immunotherapy with ISS-ODN in subjects with allergic asthma, significantly increased expression of IFN- $\gamma$, monokine induced by IFN- $\gamma$ (MIG), IFN-stimulated gene (ISG)-54, monocyte chemotactic protein (MCP)-1 and MCP-2 [67]. Also, the treatment of patients with

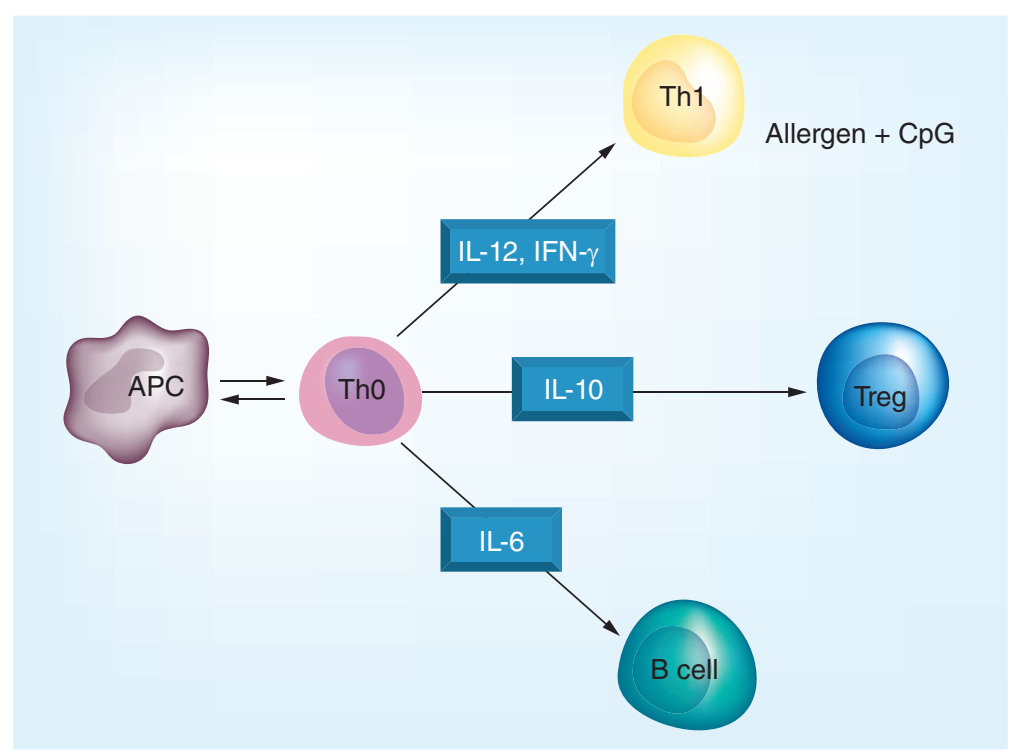

Figure 2. Co-administration of CpG and allergen induces Th1 and regulatory $\mathrm{T}$ and $\mathrm{B}$ cells, which inhibit Th2 cells.

APC: Antigen-presenting cell; Th: Helper T cell; T reg: Regulatory T cell. 
allergic asthma by QbG10 was found to be effective in the control of asthma. QbG10 is a novel TLR9 agonist packaged into virus-like particles that stimulates the immune system toward a Th1 immune response [68]. Additionally, immunotherapy with a house dust mite allergen extract along with A-type CpG ODN packaged into virus-like particles (QbG10) reduced symptoms of $\mathrm{AR}$ and asthma [66].

\section{Other allergic disorders}

With regard to CpG-ODN immunotherapy used in the treatment of other allergic disorders, the report from an investigation on human patients with ragweed allergy showed that ISS-ODN conjugated Amb a1 expressed Th1 cytokine while down regulated Th2 cytokine in PBMCs [69]. Also, Tulic et al. demonstrated that AIC immunotherapy was effective in inhibition of allergen specific $\mathrm{Th} 2$ and induction of allergen specific Th1 responses in ragweed allergic subjects [70]. Moreover, in another study it was shown that AIC immunotherapy was a potent agent for inducing Th1 immune responses in patients with ragweed allergy [71].

In another study, Klimek et al. used nanoparticles which were loaded with TLR9 ligands (CYT003QbG10) in patients with rhinoconjunctivitis. They found that Rhinoconjunctivitis symptoms were significantly lower in patients treated with high dose CYT003-QbG10 as compared with the placebo. Therefore, treatment with CYT003-QbG10 was found to be safe and tolerable in patients suffering from allergy [72]. Also, it was shown that AIC immunotherapy in patients with ragweed allergy caused redirection of immune response toward Th1 and significantly increased IFN- $\gamma$, CXCL9, CXCL10 while decreased IL-5, CCL17 and CCL22 [73].

\section{Future perspective}

Immunotherapy with CpG-ODN could be applied by two distinct strategies: immunomodulation and vaccination. Therefore, these data suggest that CpG-ODN immunotherapy could be a novel therapeutic method for the treatment of asthma and allergic diseases.

\section{Financial \& competing interests disclosure}

The authors have no relevant affiliations or financial involvement with any organization or entity with a financial interest in or financial conflict with the subject matter or materials discussed in the manuscript. This includes employment, consultancies, honoraria, stock ownership or options, expert testimony, grants or patents received or pending, or royalties.

No writing assistance was utilized in the production of this manuscript.

\section{Executive summary}

- CpG-oligodeoxynucleotide (ODN) was used as an adjuvant for the treatment of asthma and allergic diseases in mice models and humans.

- Immunotherapy with CPG-ODN could be applied by two distinct strategies: immunomodulation and vaccination.

- CPG-ODN vaccination is allergen specific and has a long lasting effect on immune system rather than immunomodulation. Both methods are effective in the prevention and treatment of allergic disease in murine models and clinical trials.

- Immunotherapy with CpG-ODN decreases eosinophilic inflammation, Th2 responses, IgE levels and Th2 cytokines expression including IL-4 and IL-5. On the contrary, it increases Th1 cell responses, cytokine secretion of IFN- $\gamma$, IL-6, IL-12 and induces regulatory T cells. Moreover, CpG-ODN immunotherapy by induction of indoleamine 2,3-dioxygenase suppresses Th2-mediated responses in the murine models of allergic diseases.

\section{References}

1 Howarth PH. Is allergy increasing? Early life influences. Clin. Exp. Allergy 28(Suppl. 6), 2-7 (1998).

2 Weiss ST. Eat dirt - the hygiene hypothesis and allergic diseases. N. Engl. J. Med. 347(12), 930-931 (2002).

3 Kay AB. Overview of 'allergy and allergic diseases: with a view to the future'. Br. Med. Bull. 56(4), 843-864 (2000).

4 Sewell WA, Thomas WR. Immunotherapy of allergic diseases by bacterial products. Immunol. Cell Biol. 89(7), 749-750 (2011).

5 Venarske D, Deshazo RD. Molecular mechanisms of allergic disease. South Med. J. 96(11), 1049-1054 (2003).

6 Gangloff SC, Guenounou M. Toll-like receptors and immune response in allergic disease. Clin. Rev. Allergy Immunol. 26(2), 115-125 (2004).

7 Prescott SL. Allergy takes its toll: the role of Toll-like receptors in allergy pathogenesis. World Allergy Organ J. 1(1), 4-8 (2008).

8 Dabbagh K, Lewis DB. Toll-like receptors and T-helper-1/Thelper-2 responses. Curr. Opin. Infect. Dis. 16(3), 199-204 (2003).

9 Feleszko W, Jaworska J, Hamelmann E. Toll-like receptors - novel targets in allergic airway disease (probiotics, friends and relatives). Eur. J. Pharmacol. 533(1-3), 308-318 (2006).

10 Akira S. TLR signaling. Curr. Top Microbiol. Immunol. 311, 1-16 (2006).

11 Ulevitch RJ. Therapeutics targeting the innate immune 
system. Nat. Rev. Immunol. 4(7), 512-520 (2004). receptors. Int. Immunopharmacol. 6(6), 863-869 (2006).

13 Bauer S, Hangel D, Yu P. Immunobiology of toll-like receptors in allergic disease. Immunobiology 212(6), 521-533 (2007).

14 Kawai T, Akira S. TLR signaling. Cell. Death Differ. 13(5), $816-825$ (2006).

15 Takeuchi O, Hoshino K, Kawai T et al. Differential roles of TLR2 and TLR4 in recognition of Gram-negative and Gram-positive bacterial cell wall components. Immunity 11(4), 443-451 (1999).

16 Takeuchi O, Kaufmann A, Grote K et al. Cutting edge: preferentially the R-stereoisomer of the mycoplasmal lipopeptide macrophage-activating lipopeptide- 2 activates immune cells through a Toll-like receptor 2- and MyD88dependent signaling pathway. J. Immunol. 164(2), 554-557 (2000)

17 Takeuchi O, Sato S, Horiuchi T et al. Cutting edge: role of Toll-like receptor 1 in mediating immune response to microbial lipoproteins. J. Immunol. 169(1), 10-14 (2002).

18 Alexopoulou L, Holt AC, Medzhitov R, Flavell RA. Recognition of double-stranded RNA and activation of NFkappaB by Toll-like receptor 3. Nature 413(6857), 732-738 (2001).

19 Hemmi H, Takeuchi O, Kawai T et al. A Toll-like receptor recognizes bacterial DNA. Nature 408(6813), 740-745 (2000)

20 Coban C, Ishii KJ, Kawai T et al. Toll-like receptor 9 mediates innate immune activation by the malaria pigment hemozoin. J. Exp. Med. 201(1), 19-25 (2005).

21 Jurk M, Heil F, Vollmer J et al. Human TLR7 or TLR8 independently confer responsiveness to the antiviral compound R-848. Nat. Immunol. 3(6), 499 (2002).

22 Hoshino K, Takeuchi O, Kawai T et al. Pillars article: cutting edge: Toll-like receptor 4 (TLR4)-Deficient mice are hyporesponsive to lipopolysaccharide: evidence for TLR4 as the Lps gene product. J. Immunol. 197(7), 2563-2566 (2016).

23 Hayashi F, Smith KD, Ozinsky A et al. The innate immune response to bacterial flagellin is mediated by Toll-like receptor 5. Nature 410(6832), 1099-1103 (2001).

24 Gereda JE, Leung DY, Thatayatikom A et al. Relation between house-dust endotoxin exposure, type $1 \mathrm{~T}$-cell development, and allergen sensitisation in infants at high risk of asthma. Lancet 355(9216), 1680-1683 (2000).

25 Horner AA, Raz E. Do microbes influence the pathogenesis of allergic diseases? Building the case for Toll-like receptor ligands. Curr. Opin. Immunol. 15(6), 614-619 (2003).

26 Fonseca DE, Kline JN. Use of CpG oligonucleotides in treatment of asthma and allergic disease. Adv. Drug Deliv. Rev. 61(3), 256-262 (2009).

27 Takeda K, Kaisho T, Akira S. Toll-like receptors. Annu. Rev. Immunol. 21 335-376 (2003)

Latz E, Schoenemeyer A, Visintin A et al. TLR9 signals after translocating from the ER to CpG DNA in the lysosome. Nat. Immunol. 5(2), 190-198 (2004).
29 Kumagai Y, Takeuchi O, Akira S. TLR9 as a key receptor for the recognition of DNA. Adv. Drug Deliv. Rev. 60 (7), 795-804 (2008).

30 Krieg AM. CpG motifs in bacterial DNA and their immune effects. Annu. Rev. Immunol. 20, 709-760 (2002).

31 Silverman ES, Drazen JM. Immunostimulatory DNA for asthma: better than eating dirt. Am. J. Respir. Cell Mol. Biol. 28(6), 645-647 (2003).

32 Klinman D, Shirota H, Tross D, Sato T, Klaschik S. Synthetic oligonucleotides as modulators of inflammation. J. Leukoc. Biol. 84(4), 958-964 (2008).

33 Krieg AM. Antiinfective applications of Toll-like receptor 9 agonists. Proc. Am. Thorac. Soc. 4(3), 289-294 (2007).

34 Horner AA, Raz E. Immunostimulatory sequence oligodeoxynucleotide-based vaccination and immunomodulation: two unique but complementary strategies for the treatment of allergic diseases. J. Allergy Clin. Immunol. 110(5), 706-712 (2002).

35 Vollmer J, Krieg AM. Immunotherapeutic applications of CpG oligodeoxynucleotide TLR9 agonists. Adv. Drug Deliv. Rev. 61(3), 195-204 (2009).

36 Krieg AM. Therapeutic potential of Toll-like receptor 9 activation. Nat. Rev. Drug Discov. 5(6), 471-484 (2006).

37 Wilson HL, Dar A, Napper SK, Marianela Lopez A, Babiuk LA, Mutwiri GK. Immune mechanisms and therapeutic potential of $\mathrm{CpG}$ oligodeoxynucleotides. Int. Rev. Immunol. 25(3-4), 183-213 (2006).

38 Krug A, Rothenfusser S, Hornung $\mathrm{V}$ et al. Identification of $\mathrm{CpG}$ oligonucleotide sequences with high induction of IFNalpha/beta in plasmacytoid dendritic cells. Eur. J. Immunol. 31(7), 2154-2163 (2001).

39 Hartmann G, Battiany J, Poeck H et al. Rational design of new $\mathrm{CpG}$ oligonucleotides that combine B cell activation with high IFN-alpha induction in plasmacytoid dendritic cells. Eur. J. Immunol. 33(6), 1633-1641 (2003).

40 Lipford GB, Bendigs S, Heeg K, Wagner H. Poly-guanosine motifs costimulate antigen-reactive CD8 T cells while bacterial CpG-DNA affect T-cell activation via antigenpresenting cell-derived cytokines. Immunology 101(1), 46-52 (2000).

41 Takeda K, Akira S. TLR signaling pathways. Semin. Immunol. 16(1), 3-9 (2004).

42 Kawagoe T, Sato S, Jung A et al. Essential role of IRAK-4 protein and its kinase activity in Toll-like receptor-mediated immune responses but not in TCR signaling. J. Exp. Med. 204(5), 1013-1024 (2007).

43 Uematsu S, Akira S. Toll-like receptors and innate immunity. J. Mol. Med. (Berl.) 84(9), 712-725 (2006).

44 Kawai T, Akira S. The role of pattern-recognition receptors in innate immunity: update on Toll-like receptors. Nat. Immunol. 11(5), 373-384 (2010).

45 Horner AA, Van Uden JH, Zubeldia JM, Broide D, Raz E. DNA-based immunotherapeutics for the treatment of allergic disease. Immunol. Rev. 179 102-118 (2001). Hayashi T, Raz E. TLR9-based immunotherapy for allergic disease. Am. J. Med. 119(10), 897 e891-896 (2006). 
47 Horner AA, Nguyen MD, Ronaghy A, Cinman N, Verbeek $S$, Raz E. DNA-based vaccination reduces the risk of lethal anaphylactic hypersensitivity in mice. J. Allergy Clin. Immunol. 106(2), 349-356 (2000).

48 Shirota H, Sano K, Hirasawa N et al. Novel roles of CpG oligodeoxynucleotides as a leader for the sampling and presentation of $\mathrm{CpG}$-tagged antigen by dendritic cells. J. Immunol. 167(1), 66-74 (2001).

49 Jarnicki AG, Conroy H, Brereton C et al. Attenuating regulatory $\mathrm{T}$ cell induction by TLR agonists through inhibition of p38 MAPK signaling in dendritic cells enhances their efficacy as vaccine adjuvants and cancer immunotherapeutics. J. Immunol. 180(6), 3797-3806 (2008).

50 Kline JN. Eat dirt: CpG DNA and immunomodulation of asthma. Proc. Am. Thorac Soc. 4(3), 283-288 (2007).

51 Hayashi T, Beck L, Rossetto C et al. Inhibition of experimental asthma by indoleamine 2,3-dioxygenase. J. Clin. Invest. 114(2), 270-279 (2004).

52 Hussain I, Jain VV, Kitagaki K, Businga TR, O’shaughnessy $\mathrm{P}$, Kline JN. Modulation of murine allergic rhinosinusitis by $\mathrm{CpG}$ oligodeoxynucleotides. Laryngoscope 112(10), 1819-1826 (2002).

53 Rhee CS, Libet L, Chisholm D et al. Allergen-independent immunostimulatory sequence oligodeoxynucleotide therapy attenuates experimental allergic rhinitis. Immunology 113(1), 106-113 (2004).

54 Kline JN, Waldschmidt TJ, Businga TR et al. Modulation of airway inflammation by $\mathrm{CpG}$ oligodeoxynucleotides in a murine model of asthma. J. Immunol. 160(6), 2555-2559 (1998).

55 Sur S, Wild JS, Choudhury BK, Sur N, Alam R, Klinman DM. Long term prevention of allergic lung inflammation in a mouse model of asthma by CpG oligodeoxynucleotides. J. Immunol. 162(10), 6284-6293 (1999).

56 Santeliz JV, Van Nest G, Traquina P, Larsen E, Wills-Karp M. Amb a 1-linked CpG oligodeoxynucleotides reverse established airway hyperresponsiveness in a murine model of asthma. J. Allergy Clin. Immunol. 109(3), 455-462 (2002).

57 Shirota H, Sano K, Kikuchi T, Tamura G, Shirato K. Regulation of murine airway eosinophilia and Th2 cells by antigen-conjugated $\mathrm{CpG}$ oligodeoxynucleotides as a novel antigen-specific immunomodulator. J. Immunol. 164(11), 5575-5582 (2000).

58 Magone MT, Chan CC, Beck L, Whitcup SM, Raz E. Systemic or mucosal administration of immunostimulatory DNA inhibits early and late phases of murine allergic conjunctivitis. Eur. J. Immunol. 30 (7), 1841-1850 (2000).

59 Cho JY, Miller M, Baek KJ et al. Immunostimulatory DNA inhibits transforming growth factor-beta expression and airway remodeling. Am. J. Respir. Cell Mol. Biol. 30(5), 651-661 (2004).

60 Fanucchi MV, Schelegle ES, Baker GL et al. Immunostimulatory oligonucleotides attenuate airways remodeling in allergic monkeys. Am. J. Respir. Crit. Care Med. 170(11), 1153-1157 (2004).
61 San Roman B, Irache JM, Gomez S, Gamazo C, Espuelas S. Co-delivery of ovalbumin and $\mathrm{CpG}$ motifs into microparticles protected sensitized mice from anaphylaxis. Int. Arch. Allergy Immunol. 149(2), 111-118 (2009).

62 Creticos PS, Schroeder JT, Hamilton RG et al. Immunotherapy with a ragweed-toll-like receptor 9 agonist vaccine for allergic rhinitis. N. Engl. J. Med. 355(14), 1445-1455 (2006).

63 Asai K, Foley SC, Sumi Y et al. Amb a 1-immunostimulatory oligodeoxynucleotide conjugate immunotherapy increases CD $4+C D 25+T$ cells in the nasal mucosa of subjects with allergic rhinitis. Allergol. Int. 57(4), 377-381 (2008).

64 Farrokhi S, Mousavi T, Arshi S, Varasteh A, Rezaei N, Salekmoghadam A. Co-administration of chenopodium album allergens and $\mathrm{CpG}$ oligodeoxynucleotides effects on peripheral blood mononuclear cells of patients with Allergic Rhinitis treated with intranasal corticosteroids and antihistamines. Iran J. Allergy Asthma Immunol. 10(2), 101-110 (2011).

65 Tulic MK, Fiset PO, Christodoulopoulos P et al. Amb a 1-immunostimulatory oligodeoxynucleotide conjugate immunotherapy decreases the nasal inflammatory response. J. Allergy Clin. Immunol. 113(2), 235-241 (2004).

66 Senti G, Johansen P, Haug S et al. Use of A-type CpG oligodeoxynucleotides as an adjuvant in allergen-specific immunotherapy in humans: a Phase I/IIa clinical trial. Clin. Exp. Allergy 39(4), 562-570 (2009).

67 Gauvreau GM, Hessel EM, Boulet LP, Coffman RL, O'byrne PM. Immunostimulatory sequences regulate interferon-inducible genes but not allergic airway responses. Am. J. Respir. Crit. Care Med. 174(1), 15-20 (2006).

68 Beeh KM, Kanniess F, Wagner F et al. The novel TLR-9 agonist QbG10 shows clinical efficacy in persistent allergic asthma. J. Allergy Clin. Immunol. 131(3), 866-874 (2013).

69 Marshall JD, Abtahi S, Eiden JJ et al. Immunostimulatory sequence DNA linked to the Amb a 1 allergen promotes $\mathrm{T}(\mathrm{H}) 1$ cytokine expression while downregulating $\mathrm{T}(\mathrm{h}) 2$ cytokine expression in PBMCs from human patients with ragweed allergy. J. Allergy Clin. Immunol. 108(2), 191-197 (2001).

70 Tulic MK, Christodoulopoulos P, Fiset PO et al. Local induction of a specific Th1 immune response by allergen linked immunostimulatory DNA in the nasal explants of ragweed-allergic subjects. Allergol Int. 58(4), 565-572 (2009).

71 Spiegelberg HL, Raz E. DNA-based approaches to the treatment of allergies. Curr Opin. Mol. Ther. 4(1), 64-71 (2002).

72 Klimek L, Willers J, Hammann-Haenni A et al. Assessment of clinical efficacy of CYT003-QbG10 in patients with allergic rhinoconjunctivitis: a Phase IIb study. Clin. Exp. Allergy 41(9), 1305-1312 (2011).

73 Simons FE, Shikishima Y, Van Nest G, Eiden JJ, Hayglass KT. Selective immune redirection in humans with ragweed allergy by injecting Amb a 1 linked to immunostimulatory DNA. J. Allergy Clin. Immunol. 113(6), 1144-1151 (2004). 